\title{
São os membros superiores na Paralisia Cerebral negligenciados?
}

\author{
Are upper limbs in cerebral paralysis: neglected?
}

\author{
Ubiratan Brum Castro ${ }^{1}$, Lúcio Honório de Carvalho Júnior², Patrícia Maria de Moraes Barros Fucs ${ }^{3}$ \\ 1. Professor Adjunto da Universidade Federal de Minas Gerais, Brasil. 2. Professor Associado da Universidade Federal de Minas Gerais, Brasil. \\ 3. Professora Associada da Faculdade de Ciências Médicas da Santa Casa de São Paulo, Brasil.
}

\begin{abstract}
Introduction: the treatment of patients with cerebral palsy aims to make it independent and efficient in their activities, despite their deficiencies. Objective: the purpose of this essay-paper is to assess whether, in a specialized service, the upper limbs are deprecated or overlooked in the treatment of motor changes of Cerebral Palsy. Methods: we analyzed 80 patients with Cerebral Palsy, under the age of seventeen years old handled institutionally. They had been treated by the same examiner regarding the classification and treatment (surgical or not) of the locomotive system. Results: the upper limbs had the following therapeutic approach considering the Group of jeopardized youngsters. One (2\%) was submitted to a tendon transfer into his wrist and 53 (98\%) were not operated on. As for Orthotics, nine (17\%) used wrist stabilizers and 45 (83\%) did not use them. As for botulinum toxin, there was no application of it in the upper limbs. In the lower limbs, considering the total number of patients, $16(20 \%)$ underwent surgery and $64(80 \%)$ were not operated on. The surgeries were: a surgical treatment of hip dislocation, seven multiple stretches in the lower limbs, five stretches of three tenotomias of Achilles tendon adductors. Regarding the use of Orthotics, 49 (61\%) made use of them and $31(39 \%)$ did not use them. Of all patients who made use of Orthotics, 48 used stabilizing ankle splints and one wore a knee stabilizer. Regarding the use of botulinum toxin: 32 had applications (40\%) and 48 received no application of it (60\%). Conclusion: in this study, the treatment of the upper limbs was neglected.
\end{abstract}

Key-words: Cerebral Palsy. Upper Limb. Treatment.

\section{Resumo}

Introdução: o tratamento do paciente com paralisia cerebral tem o objetivo de torná-lo independente e eficiente em suas atividades, a despeito de suas deficiências. Objetivo: avaliar se, em um serviço de atendimento especializado, os membros superiores são preteridos ou negligenciados no tratamento das alterações motoras da Paralisia Cerebral. Métodos: foram analisados 80 pacientes portadores de Paralisia Cerebral, menores de dezessete anos tratados institucionalmente. Foram avaliados pelo mesmo examinador quanto à classificação e tratamentos (cirúrgico ou não) do aparelho locomotor. Resultados: os membros superiores tiveram a seguinte abordagem terapêutica considerando o grupo que apresentava comprometimento de uma das mãos: um (2\%) foi submetido à transferência tendinosa no punho, e 53 (98\%) não foram operados. Quanto a órteses, nove $(17 \%)$ usavam estabilizadores de punho e $45(83 \%)$ não usavam. Quanto à toxina botulínica, não houve aplicação nos membros superiores. Nos membros inferiores, considerando o total de pacientes, 16 (20\%) foram submetidos à cirurgia e 64 (80\%) não foram operados. As cirurgias foram: um tratamento cirúrgico de luxação do quadril, sete alongamentos múltiplos em membros inferiores, cinco alongamentos de tendão calcâneo, três tenotomias de adutores. Quanto ao uso de órteses, 49 (61\%) faziam uso e 31(39\%) não as utilizavam. Dos pacientes que faziam uso de órteses, 48 usavam tala estabilizadora de tornozelo e um usava estabilizador de joelho. Quanto ao o uso de toxina botulínica: 32 tiveram aplicação (40\%) e 48 não receberam aplicação (60\%). Conclusão: no presente estudo, o tratamento do membro superior foi negligenciado.

Palavras Chave: Paralisia Cerebral. Membro Superior. Tratamento.

\section{INTRODUÇÃO}

A Paralisia Cerebral (PC) "descreve um grupo de desordens permanentes do desenvolvimento do movimento e da postura que causa limitação das atividades e são atribuídas a distúrbios não progressivos que ocorreram no desenvolvimento fetal ou no cérebro infantil"1.

O tratamento do paciente com PC visa torná-lo independente e eficiente em suas atividades a despeito de suas deficiências, melhorando sua comunicação, seu desempenho em atividades da vida diária (AVD), com melhora de sua mobilidade e de sua capacidade de marcha.

O tratamento das alterações motoras pode ser conservador (reabilitação, uso de órteses, farmacológico) cirúrgico, ou pela combinação desses meios.

Correspondência: Ubiratan Brum Castro. Professor adjunto de Ortopedia e Traumatologia, Paralisia cerebral e Cirurgia da mão da Universidade Federal de Minas Gerais. E-mail: ubrum@terra.com.br

Conflito de interesses: Os autores declaram não ter conflito de interesses.

Recebido em 29 Abr 2014; Revisado em 7 Mai 2014; Aceito em 5 Jun 2014. 
Alterações estéticas e funcionais são motivações para intervenções nos membros superiores. Elas têm como objetivo principal adequar suas funções para atividades da vida diária ainda que o paciente não consiga deambular.

Se, no tratamento dos distúrbios dos membros superiores na PC, não há dúvidas quanto à importância da reabilitação física precoce e frequente, não existe a mesma certeza quanto ao uso de órteses e quanto ao uso de agentes farmacológicos em casos selecionados. A mesma incerteza existe quanto ao tratamento cirúrgico que, historicamente, é mais indicado para correções nos membros inferiores do que nos superiores ${ }^{2}$.

Este estudo tem como objetivo avaliar o tratamento dos membros superiores em um serviço de atendimento especializado em pacientes com PC.

\section{MÉTODOS}

Realizou-se estudo observacional, transversal, não controlado, de 80 pacientes portadores de PC matriculados na Associação de Pais e Amigos dos Excepcionais (APAE) de Juiz de Fora - MG, no ano de 2007. Todos recebem tratamento multiprofissional nessa entidade desde o nascimento.

O projeto de pesquisa foi aprovado pela Comissão de Ética em Pesquisa em Seres Humanos da Irmandade da Santa Casa de Misericórdia de São Paulo sob o número 271/05.

Todos os pacientes foram avaliados pelo mesmo médico ortopedista quanto à classificação e tratamentos (cirúrgico ou não) do aparelho locomotor. A capacidade funcional do membro superior foi avaliada a partir da classificação do Committee on Spastic Hand Evaluation ${ }^{2}$.

Todos os pacientes selecionados para estudo foram incluídos, mesmos os com deficiência cognitiva grave.

\section{RESULTADOS}

Os sujeitos da pesquisa eram 36 do sexo masculino (45\%) e 44 do sexo feminino (55\%). A idade dos pacientes variou de dois a 17 anos, com média de 9,6 anos. Quanto ao diagnóstico do tipo de manifestação, 15 eram espásticos hemiplégicos (18,7\%), 15 espásticos diplégicos $(18,7 \%), 35$ espásticos quadriplégicos $(43,8 \%), 8$ discinéticos $(10 \%), 3$ atáxicos (3,8\%), 2 hipotônicos (2,5\%) e 2 mistos (2,5\%).

Os pacientes foram classificados quanto à função do membro superior, segundo o Committee on Spastic Hand Evaluation: 25 foram considerados excelentes (32.5\%), 14 bons $(17,5 \%), 18$ classificados como satisfatórios $(22,5 \%)$ e 22 considerados como maus $(27,5 \%)$, conforme apresentação na Tabela 1.
Tabela 1. Classificação dos pacientes com Paralisia Cerebral, Comitee on Spastic Hand Evaluation - APAE/JF - 2004.

\begin{tabular}{cc}
\hline Classificação & Percentual \\
\hline Excelente & 32,5 \\
Bom & 17,5 \\
Satisfatório & 22,5 \\
Mau & 27,5 \\
& \\
\hline Total & 100 \\
\hline
\end{tabular}

Fonte: Prontuário Médico - APAE/JF

Entre os 80 pacientes avaliados, $26(32,5 \%)$ tinham mãos classificadas como excelentes, sem comprometimento, e 54 $(67,5 \%)$ foram classificadas com algum comprometimento funcional do membro superior.

A abordagem terapêutica no membro superior foi: Um paciente $(2 \%)$ foi submetido à transferência tendinosa no punho e 53 (98\%) não foram operados. Quanto ao uso de órteses, nove (17\%) usavam estabilizadores de punho e 45 $(83 \%)$ não usavam. Quanto à toxina botulínica não houve aplicação nos membros superiores, sendo indicação dos aplicadores apenas para os membros inferiores.

Nos membros inferiores, considerando o total de pacientes, $16(20 \%)$ foram submetidos a cirurgias e $64(80 \%)$ não foram operados. As cirurgias foram: um caso de tratamento cirúrgico de luxação do quadril, sete casos de alongamentos múltiplos em membros inferiores, cinco casos de alongamento de tendão calcâneo, três casos de tenotomia de adutores.

Quanto ao uso de órteses nos membros inferiores, 49 (61\%) faziam uso e 31 (39\%) não a utilizavam. Dos pacientes que faziam uso de órteses, 48 usavam tala estabilizadora de tornozelo e um usava estabilizador de joelho.

Quanto ao uso de toxina botulínica, 32 pacientes tiveram aplicação (40\%) e 48 não a receberam (60\%).

\section{DISCUSSÃO}

A classificação do comprometimento da PC varia com o tipo e a localização da alteração motora. Ainda assim, essa classificação está sujeita a graduações de acometimento e independência para atividades da vida diária.

O membro superior é avaliado por classificações que buscam identificar o grau de função manual. A classificação usada nesse estudo mostra grande número de pacientes com algum comprometimento dos membros superiores. Esse quadro é frequente em hemiplégicos ${ }^{3}$. Essa é uma classificação que serve para avaliar o potencial de utilização da mão e a viabilidade de tratamento com resultados funcionais. 
Houve diferença significativa quanto ao tratamento cirúrgico ou conservador com uso de órtese ou toxina botulínica entre o membro superior e o membro inferior.

Skoff et $a l^{2}$ afirmam que, historicamente, a maior ênfase tem sido direcionada para se conseguir o pé plantígrado, a cabeça do fêmur anatomicamente localizada e a coluna retificada. Quanto à menor indicação de cirurgia nos membros superiores, as razões podem ser associadas aos resultados desses procedimentos. Carrol ${ }^{5}$ afirma que os resultados são mais cosméticos que funcionais. Skoff et $a l^{2}$, Goldner et $a l^{6}$ e Samilson et $a l^{7}$, afirmam que os resultados são muito ruins. Carlson encontrou indicação de procedimentos cirúrgicos em $50 \%$ de pacientes, seja para melhor funcionalidade, seja para melhora da deformidade ou da higiene ${ }^{8}$.

A pouca indicação de órtese para os membros superiores quando comparada aos membros inferiores ampara-se na avaliação dos familiares de que tal órtese atrapalha o movimento quando o indivíduo está acordado, e atrapalha o sono quando este está dormindo. Nos membros inferiores, melhora a qualidade da locomoção, assim como no desempenho de atividades da rotina diária ${ }^{9}$. Segundo AuttiRamo et al, as órteses de membro superior apresentam resultados variáveis enquanto no membro inferior há clara melhora na flexão plantar ${ }^{10}$.

Não foi identificada justificativa para o não uso da toxina botulínica nos membros superiores. A aplicação nos membros superiores é segura, pois diminui a espasticidade e melhora a qualidade de vida do paciente ${ }^{11,12}$. Os benefícios na diminuição da hipertonia muscular são iguais nos membros superiores e inferiores. Enquanto nos membros inferiores há clara melhora no padrão de marcha,(principalmente na espasticidade equinovara), nos membros superiores, ainda não há evidência suficiente de melhora funcional ${ }^{13}$.

\section{CONCLUSÕES}

O tratamento dos membros superiores na PC foi negligenciado quando comparado aos membros inferiores em relação ao uso de toxina botulínica.

\section{REFERÊNCIAS}

1. Rosembaum P, Paneth N, Leviton A, Goldstein M, Bax M, Damiano D, et al. A report: the definition and classification of cerebral palsy, April 2006. Dev Med Child Neurol. 2007Feb;Suppl 109:8-14.PubMed PMID 17370477.

2. Skoff H, Woodbury DF. Current concepts review \& management of the upper extremity in cerebral palsy. J Bone Joint Surg. 1985 Mar 01; 67(3):500-503.

3. Zancolli EA, Goldner LJ, Swanson AB. Surgery of the spastic hand in cerebral palsy: report of the Committee of Spastic Hand Evaluation. J Hand Surg.set 1983; 8(5pt 2):766-772. PubMed PMID 6630956.

4. Boyd RN, Morris ME, Graham HK. Management of upper limb dysfunction in children with cerebral palsy: a systematic review. Eur J Neur.Nov 2001; 8(Suppl5):150-166. PubMed PMID 11851744.

5. Carrol RE, Craig FS.The surgical treatment of cerebral palsy.I - The upperextremity. Surg clin North America. 1950. 31:385-390. PubMed PMID: 14817663.

6. Goldner JL. Reconstructive surgery of the hand in cerebral palsy and spastic paralysis resulting from injury to the spinal cord. J Bone Joint Surg. 1955 Dec 01; 37-A(6):1141-1153. PubMed PMID 13271461.

7. Samilson RL, Morris JM. Surgical improvement of the cerebral-palsied upper limb. J Bone Joint Surg. 1964 Sept 01; 46(6):1203-1216.
8. Carlson MG. Cerebral palsy. In: Wolfe SWC, Hotchkiss RN, Pederson WC, Kozin SH.Green'sOperativeHand Surgery.6th ed. Philadelphia: Elsevier; 2010.p.1139-1172.

9. Cury VCR, Mancini MC, Melo AP, Fonseca ST, Sampaio RF, Tirado MGA.Efeitos do uso de órtese na mobilidade funcional de crianças com paralisia cerebral.Rev bras fisioter.2006; 10(1): 67-74. DOI:http://dx.doi.org/10.1590/ S1413-35552006000100009.

10. Autti-Ramo I, Suoranta J, Antilla H, Malvimaara A, Makela M. Effectiveness of upper and lower limb casting and orthoses in children with cerebral palsy: an overview of review articles. Am J Phys Med Rehabil.2006 Jan; 85(1): 89-103.PubMed PMID 16357554.

11. Cardoso E, Rodrigues B, Lucena R, Oliviera IR, Pedreira $G$, Melo A. Botulinum toxin type $A$ for the treatment of the upper limb spasticity after stroke- a meta-analysis. Arq Neuropsiquiatr. 2005 mar; 63(1):30-33. PubMed PMID: 15830061.

12 Botelho LAA, Granero LHCM, Masiero D. A neurólise química simultânea com fenol e toxina botulínica do tipo A para o tratamento da espasticidade em 67 pacientes. Medde Reabl. 2012 maio 01; (59):20-24.

13. Lukban MB, Rosales R, Dressler D. Effectiveness of botulinum toxin a for upper and lower limb spasticity in children with cerebral palsy: a summary of evidence.J Neural Transm. 2009 Mar; 116(3): 319-31. PubMed PMID: 19142573. 